\title{
Development and Testing of a Variable Conductance Thermal Acquisition, Transport, and Switching System
}

\author{
D. C. Bugby ${ }^{\mathrm{a}}$, J. T. Farmer ${ }^{\mathrm{b}}$, and C. J. Stouffer ${ }^{\mathrm{a}}$ \\ ${ }^{a}$ ATK Space, 5050 Powder Mill Road, Beltsville, MD 20705 \\ (301) 902-4385;david.bugby@atk.com \\ (301) 902-4404;chuck.stouffer@atk.com \\ ${ }^{b}$ NASA Marshall Space Flight Center, MFSC, Huntsville, AL 35812 \\ (256) 544-3179; jeffery.t.farmer@nasa.gov
}

\begin{abstract}
This paper describes the development and testing of a scalable thermal control architecture for instruments, subsystems, or systems that must operate in severe space environments with wide variations in sink temperature. The architecture is comprised by linking one or more hot-side variable conductance heat pipes (VCHPs) in series with one or more cold-side loop heat pipes (LHPs). The VCHPs provide wide area heat acquisition, limited distance thermal transport, modest against gravity pumping, concentrated LHP startup heating, and high switching ratio variable conductance operation. The LHPs provide localized heat acquisition, long distance thermal transport, significant against gravity pumping, and high switching ratio variable conductance operation. Combining two variable conductance devices in series ensures very high switching ratio isolation from severe environments like the Earth's moon, where each lunar day spans 15 Earth days (270 K sink, with a surface-shielded/space viewing radiator) and each lunar night spans 15 Earth days (80-100 K radiative sink, depending on location). The single VCHPsingle LHP system described herein was developed to maintain thermal control of International Lunar Network (ILN) anchor node lander electronics, but it is also applicable to other variable heat rejection space missions in severe environments. The LHPVCHP system utilizes a stainless steel wire mesh wick ammonia VCHP, a Teflon wick propylene LHP, a pair of one-third square meter high $\varepsilon$ radiators (one capillary-pumped horizontal radiator and a second gravity-fed vertical radiator), a half-meter of transport distance, and a wick-bearing co-located flow regulator (CLFR) to allow operation with a hot (deactivated) radiator. The VCHP was designed with a small reservoir formed by extending the length of its stainless steel heat pipe tubing. The system was able to provide end-to-end switching ratios of 300-500 during thermal vacuum testing at ATK, including 3-5 W/K ON conductance and $0.01 \mathrm{~W} / \mathrm{K}$ OFF conductance. The test results described herein also include an in-depth analysis of VCHP condenser performance to explain VCHP switching operation in detail. Future multi-VCHP/multi-LHP thermal management system concepts that provide scalability to higher powers/longer transport lengths are also discussed in the paper.
\end{abstract}

